\title{
Autopercepção de saúde de usuários da Rede de Atenção à Saúde Auditiva
}

\author{
Self- perception of health by the user of the Hearing \\ Health Care Network
}

\author{
Ana Cristina de Oliveira Mares Guia, Andrezza Gonzalez Escarce², \\ Stela Maris Aguiar Lemos ${ }^{3}$
}

\begin{abstract}
Resumo
Introdução: A deficiência auditiva é um dos déficits sensoriais mais frequentes no mundo. O estudo da autopercepção de saúde poderá proporcionar o conhecimento do impacto dessa deficiência e avaliar medidas de promoção e de prevenção da saúde. Objetivo: Verificar a associação entre autopercepção de saúde, percepção de problemas auditivos e motivação para a busca de atendimento pelo usuário da Rede de Atenção à Saúde Auditiva. Método: Estudo observacional analítico transversal com amostra probabilística aleatória estratificada por município, composta por 228 usuários, vinculados aos Serviços de Atenção à Saúde Auditiva de Minas Gerais de duas microrregiões. Para a coleta de dados, foi utilizado roteiro semiestruturado. Na análise de associação, utilizou-se o Teste de Mann-Whitney, considerando associações estatisticamente significantes as que apresentaram valor $p \leq 0,05$. Resultados: Houve associação estatisticamente significante entre idade e tempo que tem o problema, bem como entre autopercepção de saúde e percepção de problemas auditivos. Os entrevistados que consideravam a alteração auditiva como problema de saúde tiveram chance 3,72 vezes maior de julgarem-se doentes. Conclusão: $O$ estudo revelou que considerar a alteração auditiva um problema de saúde esteve associado com maior chance de se achar doente. Mais da metade dos entrevistados procurou a rede devido a dificuldades de escutar e de entender as pessoas.
\end{abstract}

Palavras-chave: autoimagem; fonoaudiologia; serviços de saúde; transtornos da audição; autoavaliação.

\begin{abstract}
Background: Hearing impairment is one of the most common sensory deficits in the world. The study of self-perception of health can provide knowledge of the impact of this deficiency and evaluate health promotion and prevention measures. Objective: To verify the association between self-perception of health, perception of hearing problems and motivation to seek care by the user of the Hearing Health Care Network. Method: this is an observational analytic cross-sectional study with random probability sample stratified per municipality composed by 228 users connected to the Hearing Health Care Units of Minas Gerais in 34 cities of the two microregions. For data collection, a semi-structured script was used. In the analysis of association, the Mann-Whitney test was used, considering statistically significant associations those that presented value $p \leq 0.05$. Results: There was a statistically significant association between age and time that has the problem and between self-perception of health and perception of hearing problems. Respondents who considered auditory alteration as a health problem had a 3.72 higher odds of being considered patients. Conclusion: the study found that considering hearing impairment a health problem was associated with a higher odd of being considered ill. More than half of the interviewees searched the network because of difficulties in listening and understanding people. Keywords: self concept; hearing, speech, language and hearing sciences; health services; hearing disorders; self-assessment.
\end{abstract}

1 Programa de Pós-graduação Saúde da Criança e do Adolescente, Faculdade de Medicina, Universidade Federal de Minas Gerais (UFMG) - Belo Horizonte (MG), Brasil. ${ }^{2}$ Departamento de Fonoaudiologia, Faculdade de Medicina, Universidade Federal de Minas Gerais (UFMG) - Belo Horizonte (MG), Brasil.

${ }^{3}$ Programa de Pós-graduação em Ciências Fonoaudiológicas, Departamento de Fonoaudiologia, Faculdade de Medicina, Universidade Federal de Minas Gerais (UFMG) - Belo Horizonte (MG), Brasil.

Trabalho realizado no Programa de Pós-graduação em Ciências Fonoaudiológicas, Faculdade de Medicina, Universidade Federal de Minas Gerais (UFMG) - Belo Horizonte (MG), Brasil.

Endereço para correspondência: Ana Cristina de Oliveira Mares Guia - Rua Safira, 246 - Prado - CEP: 30411-127 - Belo Horizonte (MG), Brasil Email: anacristinamaresguia@gmail.com

Fonte de financiamento: Fundação de Amparo à Pesquisa do Estado de Minas Gerais - FAPEMIG - Edital PPSUS MS/CNPq/FAPEMIG/SES 2008-2009. Conflito de interesses: nada a declarar. 


\section{INTRODUÇÃO}

A audição é essencial para a aquisição e o desenvolvimento da linguagem. A criança com problema auditivo poderá ter um atraso no desenvolvimento da fala, da linguagem e de suas habilidades cognitivas. Já o adulto poderá sofrer alguns problemas, como dificuldades de comunicação, isolamento social e depressão, que tendem a afetar sua qualidade de vida e a dos que convivem com ele diariamente ${ }^{1}$.

No mundo, a deficiência auditiva é um dos déficits sensoriais mais frequentes. Segundo estimativa da Organização Mundial de Saúde (OMS), em 2011², cerca de 360 milhões de indivíduos apresentavam perda auditiva. Desse total, 165 milhões são adultos com idade em torno de 65 anos, 32 milhões são jovens com idade inferior a 15 anos, $80 \%$ vivem em países em desenvolvimento e cerca de $50 \%$ das perdas auditivas observadas poderiam ser evitadas com a prevenção, o diagnóstico precoce e o tratamento.

Segundo o Instituto Brasileiro de Geografia e Estatística (IBGE) ${ }^{3}$, em 2010, 45.606.648 milhões de brasileiros apresentavam algum tipo das deficiências investigadas (visual, motora, auditiva ou intelectual), dos quais 344 mil se declararam surdos, 1,8 milhão relatou grande dificuldade para ouvir e 7,5 milhões afirmaram ter alguma dificuldade auditiva. $\mathrm{O} \mathrm{IBGE}^{3}$ descreve deficiência auditiva como sendo a incapacidade auditiva, mesmo com o uso de aparelho de amplificação sonora, se a pessoa usá-lo. Além disso, o $\mathrm{IBGE}^{3}$ classifica a deficiência auditiva em: incapacidade de ouvir (pessoa que se declara totalmente surda); grande dificuldade permanente de ouvir (pessoa que declara ter grande dificuldade permanente de ouvir, mesmo usando aparelho auditivo); e alguma dificuldade permanente de ouvir (pessoa que declara ter alguma dificuldade permanente de ouvir, mesmo utilizando aparelho auditivo).

A autoavaliação do estado de saúde é um dos indicadores recomendados pela OMS para avaliar a saúde das populações. É um indicador confiável e válido das condições reais e objetivas da saúde das pessoas, representando aspectos da saúde física, cognitiva e emocional ${ }^{4}$.

A autopercepção da saúde possibilita verificar os valores e o significado atribuídos pelas pessoas à sua saúde, por meio de experiências e estados precários de saúde no contexto da vida diária. Estimar a prevalência da perda auditiva referida e a associação dessa deficiência com o estado de saúde, suas implicações na vida diária e o uso dos serviços de saúde representa uma possível redução das implicações na qualidade de vida dessa população, por intermédio do desenvolvimento e da avaliação de medidas de promoção e prevenção da saúde ${ }^{5}$.

A autopercepção de saúde é considerada um excelente indicador do estado de saúde, uma vez que extrapola a avaliação biológica do indivíduo e abrange também aspectos psicológicos e sociais, trazendo, assim, uma visão global do indivíduo ${ }^{6}$.

O presente estudo tem como objetivo verificar a associação entre autopercepção de saúde, percepção de problemas auditivos e motivação para busca de atendimento pelo usuário da Rede de Atenção à Saúde Auditiva (RASA).

\section{MÉTODO}

Trata-se de estudo observacional analítico do tipo transversal com amostra probabilística aleatória estratificada proporcional por município, composta por 228 usuários atendidos em 34 serviços de saúde auditiva, distribuídos em 34 municípios das microrregiões de Curvelo e de Sete Lagoas, Minas Gerais.

O cálculo amostral foi definido com base na produção da Rede de Saúde Auditiva das duas regiões de saúde incluídas no estudo e sua respectiva região ampliada, no período compreendido entre 2005 e 2010. Como não havia estimativas de percentuais esperados para as variáveis de interesse, foi assumido que tal percentual se situava em $50 \%$, valor que maximiza o tamanho amostral ${ }^{7}$. Foram considerados ainda um nível de confiança de 95\% e uma margem de erro de 5\%. Para definição da casuística, realizou-se o cálculo com base na produção de 3.463 Aparelhos de Amplificação Sonora Individual (AASI), no período e nas regiões citados. Tal dado quantifica o número aproximado de 1.731 pacientes, considerando adaptação bilateral. Sendo assim, a amostra final estimada foi de 228 usuários.

Para inclusão no estudo, os participantes deveriam atender aos seguintes critérios: constar nos bancos de dados do serviço de saúde auditiva, obtido na Secretaria de Estado de Saúde de Minas Gerais; ter passado pelas etapas de diagnóstico auditivo, seleção e adaptação de AASI no Sistema Único de Saúde; ter comparecido a pelo menos uma consulta no serviço de saúde auditiva nos últimos 12 meses; e ter pais ou responsáveis alfabetizados, no caso de menores de 18 anos.

Foram excluídos da pesquisa usuários com comprometimentos cognitivos, neurológicos ou psiquiátricos, relatados pelos profissionais do serviço ou acompanhante, que impedissem a compreensão das questões da entrevista.

Para a coleta dos dados, foi desenvolvido um questionário semiestruturado composto por questões que aferiram informações acerca de aspectos sociodemográficos, assistenciais e de autopercepção de saúde ${ }^{8}$. Os aspectos sociodemográficos investigados foram: idade, escolaridade, renda familiar e condições de moradia. Para investigar os aspectos assistenciais, foram incluídas duas questões: "Por que procurou a Rede de Saúde Auditiva?” (motivação para a busca do atendimento) e "Seu problema foi resolvido?". Para pesquisar a autopercepção dos usuários, foram abordadas as seguintes questões: "Você se considera doente?", "Você considera a alteração auditiva um problema de saúde?", "Há quanto tempo tem o problema de audição?”.

A aplicação do instrumento foi realizada individualmente sob a forma de entrevista por bolsistas de apoio técnico nos serviços de saúde ou nos domicílios dos participantes. Em caso de usuários menores de 12 anos, foram entrevistados apenas os 
pais ou responsáveis pelos participantes da pesquisa. No caso de sujeitos na faixa etária de 13 a 17 anos, foram entrevistados os pais ou responsáveis e o usuário do serviço, os quais assinaram o Termo de Consentimento Livre e Esclarecido e o Termo de Assentimento Livre e Esclarecido, respectivamente. Cabe ainda ressaltar que todos atenderam aos mesmos critérios de inclusão já citados.

Previamente à coleta de dados, foi realizado estudo-piloto para calibração do instrumento quanto à compreensão e ao sequenciamento das questões, ao tempo de coleta de dados e à verificação da aplicabilidade do instrumento na realidade dos serviços de saúde auditiva. $\mathrm{O}$ estudo em questão foi realizado em um serviço da Rede de Saúde Auditiva pertencente ao município de Belo Horizonte, após anuência do gestor, e seguiu os mesmos critérios adotados posteriormente na coleta de dados.

As entrevistas foram transcritas e inseridas em um banco de dados elaborado pelas pesquisadoras. As variáveis foram definidas com base nos itens presentes no questionário utilizado no estudo para a entrevista e já publicado ${ }^{7}$. Dessa forma, as variáveis de resposta adotadas foram: autopercepção de saúde, percepção de problemas auditivos e motivação para a busca de atendimento em saúde auditiva. Foram variáveis explicativas: dados sociodemográficos, tempo no serviço e idade dos usuários. A variável contínua analisada foi a idade dos usuários. Vale destacar que, em relação aos dados sociodemográficos, foram utilizadas as variáveis categóricas "Sexo" e "Escolaridade.
Foram realizadas análise descritiva da distribuição de frequência das variáveis categóricas e análise das medidas de tendência central e de dispersão para as variáveis contínuas. Inicialmente, para melhor compreensão das características da amostra, analisou-se a variável "Há quanto tempo tem o problema?", segundo as variáveis "Idade" e "Formação" (escolaridade). Para a análise de associação entre as variáveis "Você se considera doente?" "Você considera a alteração auditiva como problema de saúde?", "Por que procurou a Rede de Saúde Auditiva?” e "Seu problema foi resolvido?", utilizaram-se os testes ANOVA e do Qui-quadrado. A magnitude das associações foi aferida pelas razões de chance (Odds Ratio), com respectivos intervalos de confiança. Obtiveram-se intervalos de confiança de 95\%. Foram consideradas como associações estatisticamente significantes os resultados que apresentaram $\mathrm{p} \leq 0,05$.

Este estudo foi analisado e aprovado pelo Comitê de Ética em Pesquisa da Universidade Federal de Minas Gerais (ETIC 186-10), e todos os participantes ou seus responsáveis assinaram o Termo de Consentimento Livre e Esclarecido.

\section{RESULTADOS}

A amostra foi composta por 228 usuários, dos quais 127 do sexo feminino e 101 do sexo masculino. Pode-se verificar, na Figura 1, as informações sobre os usuários da Rede de Saúde Auditiva da região estudada.

A

B

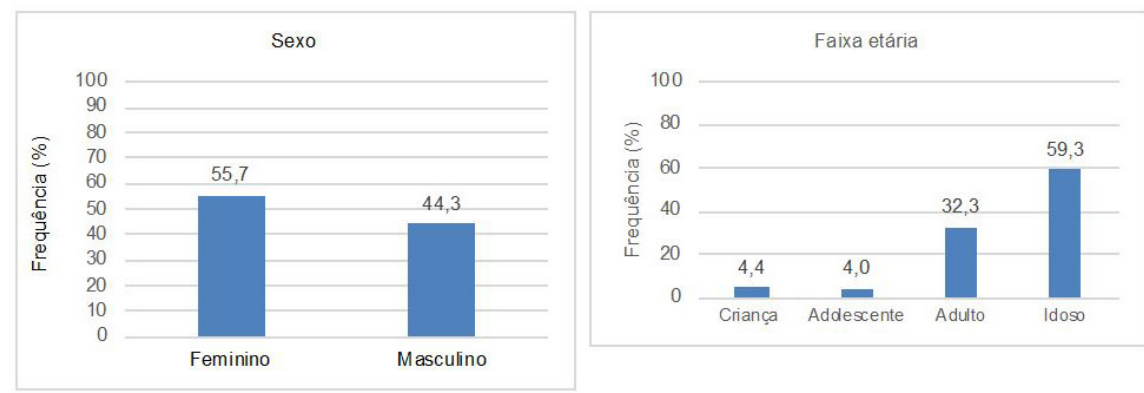

C

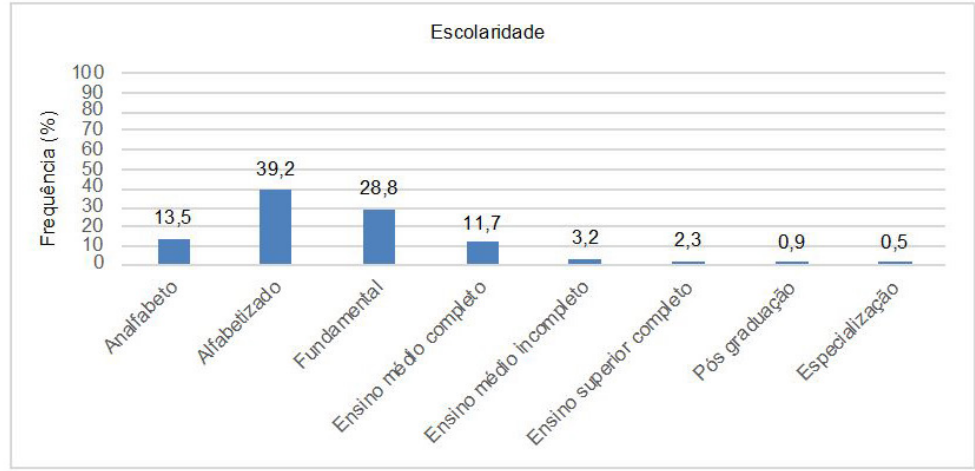

Figura 1. Gráficos de distribuição da frequência das variáveis de caracterização da amostra. Observação: Crianças abaixo da fase escolar não foram incluídas na análise escolaridade $(n=6)$ 
Em relação às características socioeconômicas dos usuários da Rede de Saúde Auditiva que responderam ao questionário, $54,6 \%$ tinham renda familiar entre 1 e 2 salários mínimos, e apenas $0,9 \%$ possuía renda superior a 8 salários mínimos; $82,8 \%$ tinham tratamento de água clorada, enquanto $7,9 \%$ não apresentavam tratamento de água; $85 \%$ recebiam abastecimento de água por meio da rede pública, ao passo que $14,5 \%$, por poços e nascentes; $18,5 \%$ não tinham saneamento básico; $100 \%$ possuíam luz elétrica; $65 \%$ não apresentavam plano de saúde privado; 90,3\% tinham casa própria (Tabela 1).

A análise da associação entre idade e tempo que tem o problema revelou diferença com significância estatística entre o grupo "Até 1 ano" e os demais grupos do estudo, conforme apresentado na Tabela 2 .

Na Tabela 3, observa-se que não houve significância estatística ( $\mathrm{p}$-valor $=0,279)$ entre escolaridade e tempo que tem o problema.

Verificou-se associação com significância estatística entre as variáveis "Você se considera doente?" e "Você considera a alteração auditiva como problema de saúde?”. Usuários que consideraram alteração auditiva como problema de saúde tiveram chance $3,72(1,95-7,09)$ vezes maior de julgarem-se doentes comparados ao grupo que não considerou a alteração auditiva como problema de saúde (Tabela 4).

Tabela 1. Distribuição da frequência das variáveis sociodemográficas. Minas Gerais - MG, 2005-2010

\begin{tabular}{|c|c|c|c|}
\hline Variável & Categorias da variável & N-válidos & $\%$ \\
\hline \multirow[t]{6}{*}{ Renda familiar } & $<$ Menor que 1 salário mínimo & 46 & 21,1 \\
\hline & Entre 1 e 2 salários mínimos & 119 & 54,6 \\
\hline & Entre 3 e 4 salários mínimos & 41 & 18,8 \\
\hline & Entre 5 e 7 salários mínimos & 10 & 4,6 \\
\hline & Maior que 8 salários mínimos & 2 & 0,9 \\
\hline & Total & 218 & 100,0 \\
\hline \multirow[t]{5}{*}{ Tratamento de água } & Filtrada & 16 & 7,0 \\
\hline & Fervida & 5 & 2,2 \\
\hline & Clorada & 188 & 82,8 \\
\hline & Sem tratamento & 18 & 8,0 \\
\hline & Total & 227 & 100,0 \\
\hline \multirow[t]{4}{*}{ bastecimento de água } & Rede pública & 193 & 85,0 \\
\hline & Poço ou nascente & 33 & 14,5 \\
\hline & Outros & 1 & 0,5 \\
\hline & Total & 227 & 100,0 \\
\hline \multirow[t]{3}{*}{ Saneamento básico } & Não & 42 & 18,5 \\
\hline & Sim & 185 & 81,5 \\
\hline & Total & 227 & 100,0 \\
\hline \multirow[t]{3}{*}{ Luz elétrica } & Não & 0 & 0,0 \\
\hline & Sim & 226 & 100,0 \\
\hline & Total & 226 & 100,0 \\
\hline \multirow[t]{3}{*}{ lano de saúde privado } & Não & 147 & 65,0 \\
\hline & Sim & 79 & 35,0 \\
\hline & Total & 226 & 100,0 \\
\hline \multirow[t]{3}{*}{ Moradia } & Casa própria & 204 & 90,3 \\
\hline & Casa alugada & 22 & 9,7 \\
\hline & Total & 226 & 100,0 \\
\hline
\end{tabular}

Tabela 2. Associação entre tempo que tem o problema e idade. Minas Gerais - MG, 2005-2010

\begin{tabular}{lccccccccc}
\multicolumn{1}{c}{\begin{tabular}{c} 
Há quanto tempo tem \\
\multicolumn{1}{c}{ o problema? }
\end{tabular}} & $\mathbf{N}$ & Média & E.P. & Mín. & $\mathbf{1}^{\circ} \mathbf{Q}$ & $\mathbf{2}^{\circ} \mathbf{Q}$ & $\mathbf{3}^{\circ} \mathbf{Q}$ & Máx. & p-Valor \\
\cline { 2 - 8 } Até 1 ano & 32 & 39,094 & 4,407 & 1 & 15,5 & 46,5 & 60,5 & 74 \\
De 1 a 3 anos & 23 & 69,652 & 3,989 & 8 & 64 & 75 & 81,5 & 92 \\
De 3 a 4 anos & 14 & 63,571 & 3,96 & 40 & 54 & 67 & 76 & 80 & $<0,001$ \\
Maior que 4 anos & 156 & 59,442 & 1,574 & 8 & 49 & 65 & 74,5 & 96 \\
\hline
\end{tabular}

Teste ANOVA. Legenda: E.P. = erro padrão; Mín. = mínimo; Máx. = máximo; $1^{\circ} \mathrm{Q}=$ primeiro quartil; $2^{\circ} \mathrm{Q}=$ segundo quartil; $3^{\circ} \mathrm{Q}=$ terceiro quartil; até 1 ano = até 11 meses e 29 dias; de 1 a 3 anos = de 12 meses e 1 dia até 2 anos, 11 meses e 29 dias; de 3 a 4 anos = de 3 anos e 1 dia até 3 anos, 11 meses e 29 dias; maior que 4 anos= a partir de 4 anos e 1 dia 
Os dados revelaram ausência de associação com significância estatística entre as variáveis "Seu problema foi resolvido?" e "Por que procurou a Rede de Saúde Auditiva?" (Tabela 5).

Vale destacar que, no item "Outros", as citações sobre a motivação para a procura pelo SASA tiveram relação com cinco aspectos: socioeconômico (não tinha dinheiro, era caro); encaminhamento de outro profissional (fonoaudiólogo, médico); encaminhamento de familiares (filhos, cônjuge); inadequação a situações funcionais, sociais ou escolares (reuniões, relacionamentos); e queixas clínicas (zumbido, coceira).

\section{DISCUSSÃO}

Este estudo investigou a autopercepção em saúde dos usuários da Rede de Saúde Auditiva das regiões de Curvelo e de Sete Lagoas, Minas Gerais, e sua relação com a percepção da alteração auditiva como problema de saúde, tendo como referência os dados da escolaridade, a renda e a qualidade de vida. Dados de qualidade de vida podem não só balizar a percepção, mas também a exposição e a vulnerabilidade a problemas auditivos.
A literatura mostra que associações com as taxas de prevalência da deficiência auditiva, devido à ocorrência de doenças de orelha média, congênita ou infecciosa e à identificação tardia de perdas auditivas de grau leve e moderado, podem ser estabelecidas com condições de vida em países em desenvolvimento ${ }^{9}$.

Em relação à variável "Sexo", o presente estudo demonstrou que houve maior utilização do serviço por mulheres, com nível de escolaridade baixo, renda mensal de até 2 salários mínimos e predominantemente idosas - tais dados também foram observados em estudos prévios ${ }^{10-12}$. Esse resultado pode ser atribuído ao fato de as mulheres apresentarem maior percepção quanto aos riscos de saúde ${ }^{13}$ e evidencia que a grande maioria dos usuários que procura o serviço de saúde auditiva tem idade superior a 60 anos. Esse dado corrobora outros estudos nacionais, feitos com usuários de AASI concedidos pelo SUS ${ }^{1,10,11}$, e reafirma dados do censo demográfico, que revelam o aumento da longevidade, o que faz com que o envelhecimento da população e a qualidade de vida nessa faixa etária sejam foco de atuação da saúde pública ${ }^{11}$.

Tabela 3. Associação entre tempo que tem o problema e escolaridade. Minas Gerais - MG, 2005-2010

\begin{tabular}{|c|c|c|c|c|c|c|c|c|c|c|}
\hline \multirow{3}{*}{ Variáveis } & \multirow{3}{*}{ Fatores } & \multicolumn{8}{|c|}{ Escolaridade } & \multirow{3}{*}{ p-valor } \\
\hline & & \multicolumn{2}{|c|}{ Analfabeto } & \multicolumn{2}{|c|}{ Alfabetizado } & \multicolumn{2}{|c|}{$\begin{array}{c}\text { Ensino médio } \\
\text { ou maior }\end{array}$} & \multicolumn{2}{|c|}{ Total } & \\
\hline & & $\mathbf{N}$ & $\%$ & $\mathbf{N}$ & $\%$ & $\mathbf{N}$ & $\%$ & $\mathbf{N}$ & $\%$ & \\
\hline \multirow{5}{*}{$\begin{array}{l}\text { Há quanto tempo } \\
\text { tem o problema? }\end{array}$} & Até 1 ano & 8 & 25 & 20 & 63 & 4 & 13 & 32 & 100 & \multirow{5}{*}{0,279} \\
\hline & De 1 a 3 anos & 4 & 17 & 17 & 74 & 2 & 9 & 23 & 100 & \\
\hline & De 3 a 4 anos & 1 & 7 & 9 & 64 & 4 & 29 & 14 & 100 & \\
\hline & Superior a 4 anos & 17 & 11 & 104 & 68 & 31 & 20 & 152 & 100 & \\
\hline & Total & 30 & 14 & 150 & 68 & 41 & 19 & 221 & 100 & \\
\hline
\end{tabular}

Tabela 4. Associação entre as variáveis "Você se considera doente?" e "Você considera a alteração auditiva como problema de saúde?". Minas Gerais - MG, 2005-2010

\begin{tabular}{|c|c|c|c|c|c|c|c|c|c|c|}
\hline \multirow{3}{*}{ Variáveis } & \multirow{3}{*}{ Fatores } & \multicolumn{6}{|c|}{ Você se considera doente? } & \multirow{3}{*}{ p-Valor } & \multirow{3}{*}{ OR } & \multirow{3}{*}{ IC } \\
\hline & & \multicolumn{2}{|c|}{ Não } & \multicolumn{2}{|c|}{ Sim } & \multicolumn{2}{|c|}{ Total } & & & \\
\hline & & $\mathbf{N}$ & $\%$ & $\mathbf{N}$ & $\%$ & $\mathbf{N}$ & $\%$ & & & \\
\hline \multirow{3}{*}{$\begin{array}{l}\text { Você considera a alteração } \\
\text { auditiva como problema de } \\
\text { saúde? }\end{array}$} & Não & 50 & $76 \%$ & 16 & $24 \%$ & 66 & $100 \%$ & \multirow{2}{*}{$<0,001$} & 1 & - \\
\hline & Sim & 73 & $46 \%$ & 87 & $54 \%$ & 160 & $100 \%$ & & 3,72 & $1,95-7,09$ \\
\hline & Total & 123 & $54 \%$ & 103 & $46 \%$ & 226 & $100 \%$ & - & - & - \\
\hline
\end{tabular}

Teste Qui-Quadrado. Legenda: OR = Odds Ratio; IC = intervalo de confiança

Tabela 5. Associação entre as variáveis "Seu problema foi resolvido?" e "Por que procurou a Rede de Saúde Auditiva?". Minas Gerais - MG, 2005-2010

\begin{tabular}{|c|c|c|c|c|c|c|c|c|c|c|}
\hline \multirow{3}{*}{ Variáveis } & \multirow{3}{*}{ Fatores } & \multicolumn{6}{|c|}{ Seu problema foi resolvido } & \multirow{3}{*}{ p-Valor } & \multirow{3}{*}{ OR } & \multirow{3}{*}{ IC } \\
\hline & & \multicolumn{2}{|c|}{ Não } & \multicolumn{2}{|c|}{ Sim } & \multicolumn{2}{|c|}{ Total } & & & \\
\hline & & $\mathbf{N}$ & $\%$ & $\mathbf{N}$ & $\%$ & $\mathbf{N}$ & $\%$ & & & \\
\hline \multirow{4}{*}{$\begin{array}{l}\text { Por que procurou } \\
\text { a Rede de Saúde } \\
\text { Auditiva? }\end{array}$} & Dificuldade para escutar & 16 & 16 & 82 & 84 & 98 & 100 & \multirow{4}{*}{0,68} & 1 & \\
\hline & Dificuldade para entender as pessoas & 10 & 20 & 39 & 80 & 49 & 100 & & 0,76 & $0,31-1,83$ \\
\hline & Outros & 7 & 23 & 24 & 77 & 31 & 100 & & 0,66 & $0,24-1,81$ \\
\hline & Total & 33 & 19 & 145 & 82 & 178 & 100 & & & \\
\hline
\end{tabular}

Teste Qui-Quadrado. Legenda: $\mathrm{OR}=$ Odds Ratio; $\mathrm{IC}$ = intervalo de confiança; $\mathrm{N}$ = número de indivíduos (varia devido a dados faltantes, pois houve usuários que não souberam/quiseram responder) 
Quanto à escolaridade, mais de 1/3 dos usuários se declarou alfabetizado, embora não tenha concluído o ensino fundamental. Esses dados corroboram estudo epidemiológico ${ }^{14}$ realizado na cidade de Guarapuava, Paraná, com idosos, no qual se observou baixo nível de escolaridade entre os entrevistados, e também dados revelados pela Pesquisa Nacional por Amostras de Domicílio (PNAD) ${ }^{15}$, no qual se verificou que $32,9 \%$ das pessoas com mais de 65 anos eram analfabetas no Brasil.

A análise dos dados dos usuários desses serviços em relação às suas idades no momento em que procuraram a RASA revelou predominância do grupo de idosos e um intervalo importante (cerca de quatro anos) entre a suspeita da perda auditiva e a procura por tratamento. Destaca-se pouca representatividade de crianças atendidas pelo serviço de saúde auditiva. Tal dado revela a necessidade do aprimoramento de programas e campanhas com orientações ao reconhecimento da perda auditiva e a importância de triagens auditivas neonatais ${ }^{11}$. Outras pesquisas também revelaram representatividade menor de crianças atendidas em serviços de diagnóstico audiológico ${ }^{11,16}$.

Reforça-se que esse dado deve ser considerado, pois pode indicar que alterações auditivas em crianças estão silentes, estão sendo negligenciadas ou não está havendo o devido acolhimento pela rede de atenção.

Por outro lado, acredita-se que a progressão da perda auditiva e o aumento das dificuldades que se relacionam a ela fazem com que os usuários adultos e idosos só procurem atendimento alguns anos após o início dos sintomas de privação auditiva ${ }^{11}$. Fatores, como a negação do problema, os papéis sociais desempenhados, a necessidade de comunicação, o acesso ao sistema para fornecimento de aparelhos auditivos, o conhecimento a respeito da deficiência auditiva, a estética, o estigma do uso do AASI e as condições de saúde, são determinantes para a procura do tratamento ${ }^{17}$.

Estudo $^{18}$ realizado na Clínica de Fonoaudiologia da Faculdade de Odontologia de Bauru, da Universidade de São Paulo, indicou que, de 106 adultos entrevistados, apenas 2,6\% procuraram atendimento no primeiro ano após o início da perda auditiva e que cerca de $50 \%$ esperaram dez anos ou mais. Outra pesquisa realizada na mesma instituição indicou que o tempo decorrido entre o início da alteração auditiva e a procura do serviço para o tratamento variou de 2 meses a 61 anos $^{17}$. Estudo ${ }^{19}$ realizado na região de Campinas, São Paulo, em duas instituições corroboram os achados da presente pesquisa.

Cabe considerar que a perda auditiva é um problema de saúde pública importante, pois a audição é um dos sentidos essenciais para a comunicação ${ }^{20}$, necessidade vital para qualquer indivíduo. O fato de o indivíduo não ouvir bem, independentemente de sua faixa etária, leva a implicações psicossociais sérias, uma vez que, com prejuízo da sua comunicação, pode ocorrer frustração, isolamento social e depressão ${ }^{21}$.
A privação auditiva ou o seu declínio durante a vida impede que o indivíduo desempenhe seu papel social de maneira plena. As crianças podem apresentar um atraso de suas habilidades linguísticas, cognitivas, sociais, emocionais e pedagógicas, enquanto os adultos ou idosos terão uma diminuição da sua capacidade de comunicação, somada a outras mudanças biológicas, psicológicas e sociais ${ }^{1}$.

Ao analisar a distribuição da variável "Há quanto tempo tem o problema?" de acordo com escolaridade, observou-se que a distribuição das respostas não foi similar. Observa-se ainda que não houve diferença estatística significativa entre os entrevistados quanto à variável "Formação". Esse dado corrobora outros estudos já realizados com pacientes adultos e idosos em serviços de audiologia ${ }^{17,18}$.

A autoavaliação do estado de saúde é uma medida de autorreferência e facilmente analisada. Apesar de ser subjetiva, pesquisadores verificaram associação com diferentes comportamentos indesejáveis à saúde e com morbidades ${ }^{22}$.

Estudo $^{23}$ realizado na cidade de Pelotas, Rio Grande do Sul, para investigação da autopercepção de saúde em adolescentes, adultos e idosos, constatou que, com o aumento da idade, há uma autorreferência pior da saúde e que os indivíduos percebem a saúde não só como ausência de doença, mas também relacionada com aspectos sociais, demográficos e comportamentais, ou seja, nível de bem-estar, nível de satisfação com a vida, capacidade funcional e qualidade de vida são importantes indicadores de autoavaliação positiva da saúde ${ }^{24,25}$.

A qualidade de vida é definida pela OMS como "a percepção do indivíduo de sua posição na vida no contexto da cultura e sistemas de valores nos quais ele vive e em relação aos seus objetivos, expectativas, padrões e preocupações”, ou seja, é uma noção eminentemente humana que se aproxima do grau de satisfação encontrado na vida familiar, social e ambiental ${ }^{26}$. Dessa forma, a qualidade de vida é um estado subjetivo de saúde, que depende da percepção de cada indivíduo e do impacto que a doença acarreta em seu estado de saúde ${ }^{26}$.

Entre as várias doenças permanentes que geram impacto e prejuízo na qualidade de vida, a deficiência auditiva deve ser destacada, pois uma alteração na audição pode gerar dificuldades na comunicação, isolamento, incapacidade de desenvolver plenamente suas funções sociais, privação do lazer, alterações psicológicas, problemas de alerta e defesa ${ }^{21}$.

No presente estudo, a chance de um entrevistado que avalia a alteração auditiva como um problema de saúde se considerar doente foi quase quatro vezes maior do que o entrevistado que não considerou a alteração auditiva como um problema de saúde. Assim, em relação à população estudada, encontrou-se alteração auditiva na percepção global de saúde.

A privação auditiva propicia o risco de incapacidades e prejuízos importantes nos diferentes ciclos de vida. Na criança, haverá prejuízo do desenvolvimento da linguagem, enquanto, no 
adulto, associado à perda da audição, ocorrerão outros problemas, como o desempenho comunicativo inadequado ${ }^{19,21}$. Os idosos podem se tornar mais reclusos e silenciosos, o que, por vezes, possibilitará uma interpretação errônea de comportamento antissocial ou mesmo início de demência ${ }^{21}$.

O déficit auditivo em idosos tem alta prevalência, mas é uma alteração pouco investigada por profissionais de saúde. É necessária uma identificação precoce da perda auditiva ou do risco de perda auditiva na atenção primária por tratar-se de um problema de início insidioso, lento e progressivo, que, muitas vezes, os pacientes não valorizam por considerarem um estado natural do envelhecimento. Já os adolescentes subestimam a dificuldade de ouvir e retardam a procura por serviços de saúde para realizar medidas de prevenção ${ }^{11,21}$. Sendo assim, a Atenção Primária à Saúde (APS) tem papel fundamental para a promoção da saúde e, portanto, para o envelhecimento saudável e o controle das multimorbidades. Ela é a ordenadora da rede de atenção e pode ser vista como uma estratégia para fortalecer o sistema de saúde. Cabe a ela a assistência permanente e o monitoramento de aspectos e patologias que interferem na qualidade de vida da população. Desse modo, medidas preventivas e parte das ações de reabilitação são responsabilidade da $\mathrm{APS}^{27}$.

Dificuldade para escutar e entender as pessoas foi citado como motivação para a busca da RASA pelos usuários do presente estudo. Assim, é possível inferir que esses usuários buscaram a rede devido ao impacto da perda auditiva na qualidade de vida.

$\mathrm{Na}$ associação entre as variáveis "Por que procurou a Rede de Saúde Auditiva?" e "Seu o problema foi resolvido?", não foi encontrada significância estatística; contudo, cerca de $80 \%$ dos usuários consideraram o seu problema sanado com a seleção e a adaptação dos aparelhos auditivos. Desde modo, identificar pessoas com deficiência auditiva precocemente e oferecer tratamento adequado trazem impacto positivo na qualidade de vida, visto que a privação auditiva é um fator importante que determina a percepção da saúde como ruim. Embora tenha sido encontrado um índice de satisfação elevado, vale lembrar que só adquirir os aparelhos não é suficiente. Há a necessidade do acompanhamento dos pacientes com a realização de terapias fonoaudiológicas visando à sua integração no processo de comunicação, no convívio social, nas dimensões psicológicas e profissionais ${ }^{28}$.

Outro estudo ${ }^{29}$ demonstra que a reabilitação auditiva propicia otimização de medidas cognitivas globais. O desenvolvimento da habilidade de atenção auditiva contribui para a diminuição do isolamento social e da dificuldade comunicativa e, portanto, para a melhora da qualidade de vida.

O presente estudo contribui para a discussão sobre a percepção do usuário da rede de atenção quanto às suas condições de saúde auditiva e às implicações na autopercepção da saúde global. Contudo, os dados apresentados não podem ser generalizados para outras regiões do país devido às características sociodemográficas e ao estágio de implantação da rede, mas podem subsidiar tanto o desenvolvimento de estudos complementares como a organização de políticas públicas.

Vale destacar que, muitas vezes, embora o usuário tenha queixa da perda de audição, o tempo transcorrido até a procura do tratamento foi superior a quatro anos. As políticas de promoção da saúde auditiva devem ser incrementadas para garantir a identificação precoce e a assistência especializada em todas as faixas etárias. Por meio dessas políticas, simples questionamentos acerca da audição poderão ser implementados e realizados já na atenção primária, garantindo uma melhor qualidade de vida à população.

Identificou-se associação entre autopercepção de saúde geral e percepção de problemas auditivos pelo usuário da Rede de Atenção à Saúde Auditiva. O estudo revelou ainda que considerar a alteração auditiva um problema de saúde esteve associado a uma chance quase quatro vezes maior de se julgar doente quando comparado a não considerar a perda auditiva como um problema de saúde. Mais da metade dos entrevistados procurou a rede devido a dificuldades de escutar e de entender pessoas. A motivação pela procura da rede ocorreu, na maioria das vezes, por queixas relacionadas ao impacto da perda auditiva.

\section{REFERÊNCIAS}

1. Buriti AKL, Oliveira SHS. Adaptação à prótese auditiva em usuários assistidos pelo Sistema Único de Saúde. Rev Soc Bras Fonoaudiol. 2012;17(1):41-6. http://dx.doi.org/10.1590/S1516-80342012000100009.

2. World Health Organization. Deafness and hearing impairment [Internet] Geneva: WHO; 2011 [citado em 2016 dez 11]. Disponível em: http://www. who.int/whosis/whostat/2011/en/

3. Instituto Brasileiro de Geografia e Estatística. Censo demográfico 2010 [Internet]. Rio de Janeiro: IBGE; 2010 [citado em 2016 dez 11]. Disponível em: http://censo2010.ibge.gov.br/

4. Pagotto V, Nakatani AYK, Silveira EA. Fatores associados à autoavaliação de saúde ruim em idosos usuários do Sistema Único. Cad Saude Publica.

2011;27(8):1593-602. http://dx.doi.org/10.1590/S0102-311X2011000800014. PMid:21877007.

5. Paiva KM. Deficiência auditiva referida e condições de saúde de idosos: um estudo de base populacional [dissertação de mestrado]. São Paulo (SP): Universidade de São Paulo. Faculdade de Saúde Pública. Departamento de Epidemiologia; 2010.

6. Confortin SC, Giehl MWC, Antes DL, Schneider IJC, d’Orsi E. Autopercepção positiva de saúde em idosos: estudo populacional no Sul do Brasil. Cad Saude Publica. 2015;31(5):1049-60. http://dx.doi.org/10.1590/0102-311X00132014. PMid:26083179.

7. Luiz RR, Magnanini MMF. A lógica da determinação do tamanho da amostra em investigações epidemiológicas. Cad Saude Colet. 2000;8(2):9-28. 
8. Guia ACOM, Oliveira R No, Escarce AG, Lemos SMA. Rede de Atenção à Saúde Auditiva: perspectiva do usuário. Dist Comum. 2016;28(3):473-82.

9. Cruz MS, Oliveira LRO, Carandina L. Inquéritos de saúde e fonoaudiologia. Rev CEFAC. 2009;11(1):166-72. http://dx.doi.org/10.1590/S151618462009005000009

10. Mondelli MFCG, Silva LSL. Perfil dos pacientes atendidos em um sistema de alta complexidade. Arq Int Otorrinol. 2011;15(1):29-34.

11. Gresele ADP, Lessa AH, Alves LC, Torres EMO, Vaucher AVA, Moraes AB, et al. Levantamento e análise de dados de pacientes atendidos em um programa de concessão de aparelhos de amplificação sonora individual. CoDAS. 2013;25(3):195-201. http://dx.doi.org/10.1590/S2317-17822013000300002. PMid:24408328.

12. Bacchetti EMS, Akiyama R, Bento RF, Samelli AG. Indicadores de qualidade para serviços de audiologia. Audiol Commun Res. 2013;18(4):268-74. http://dx.doi.org/10.1590/S2317-64312013000400007.

13. Arruda GO, Marcon SS. Inquérito sobre a utilização dos serviços de saúde por homens adultos: prevalências e fatores associados. Rev Lat Am Enfermagem. 2016;24:1-9.

14. Pilger C, Menon MH, Mathias TAF. Características sociodemográficas e de saúde de idosos: contribuições para os serviços de saúde. Rev Latino-AM Enferm. 2011;19(5):1230-8. http://dx.doi.org/10.1590/S010411692011000500022 .

15. Instituto Brasileiro de Geografia e Estatística [Internet]. Síntese de indicadores sociais - uma análise das condições de vida da população brasileira Internet]. Rio de Janeiro: IBGE; 2010 [citado em 2016 nov 14]. Disponível em: http://www.ibge.gov.br/home/estatistica/populacao/ condicaodevida/indicadoresminimos/sinteseindicsociais2010/SIS2010

16. Domingos BP. Caracterização do perfil audiológico e epidemiológico da população atendida em um programa de saúde auditiva [monografia]. Belo Horizonte: Curso de Fonoaudiologia, Universidade Federal de Minas Gerais; 2010.

17. Yanomoto CH, Ferrari VD. Relationship between hearing thresholds, handicap and the time taken to seek treatment for hearing loss. Rev Soc Bras Fonoaudiol. 2012;17(2):135-41.

18. Lima II, Aiello CP, Ferrari DV. Correlações audiométricas do questionário de handicap auditivo para adultos. Rev CEFAC. 2011;13(3):496-503. http:// dx.doi.org/10.1590/S1516-18462010005000057.
19. Sígolo C, Lacerda CBF. Da suspeita à intervenção em surdez: caracterização deste processo na região de Campinas/SP. J Soc Bras Fonoaudiol. 2011;23(1):327. http://dx.doi.org/10.1590/S2179-64912011000100009. PMid:21552730.

20. Morettin M, Cardoso MRA, Lebrão ML, Duarte YAO. Fatores relacionados à autopercepção da audição entre idosos do município de São Paulo - Projeto SABE. Saude Col. 2008;5(4):168-72.

21. Silva DPCB, Silva VB, Aurélio FS. Auditory satisfaction of patients fitted with hearing aids in the Brazilian Public Health Service and benefits offered by the hearing aids. Rev Bras Otorrinolaringol (Engl Ed). 2013;79(5):53845. http://dx.doi.org/10.5935/1808-8694.20130098. PMid:24141666.

22. Santiago LM, Novaes CO. Auto-avaliação da audição em idosos. Rev CEFAC. 2009;11(Supl 1):98-105. http://dx.doi.org/10.1590/S1516-18462009000500015.

23. Souza TF, Silva KS, Garcia LMT, Del Duca GP, Oliveira ESA, Nahas MV. Autoavaliação de saúde e fatores associados em adolescentes do Estado de Santa Catarina, Brasil. Rev Paul Pediatr. 2010;28(4):333-9. http://dx.doi. org/10.1590/S0103-05822010000400008.

24. Reichert FF, Loch MR, Capilheria MF. Autopercepção de saúde em adolescentes, adultos e idosos. Cien Saude Colet. 2012;17(12):3353-62. http://dx.doi.org/10.1590/S1413-81232012001200020. PMid:23175411.

25. Mondelli MFCG, Souza PJS. Quality of life in elderly adults before and after hearing aid fitting. Braz J Othorhinolaryngol. 2012;78(3):49-56. http:// dx.doi.org/10.1590/S1808-86942012000300010. PMid:22714847.

26. Araújo TM, Morettin M, Torres KCC, Brasil HP, Silva TA, Laperuta EB, et al Relação entre o processo de reabilitação auditiva e a auto-percepção da Qualidade de Vida em usuários de Aparelho de Amplificação Sonora Individual: revisão sistemática. Distúrb Comun. 2010;22(1):25-36.

27. Ferreira DC, Gomes EA, Souza ID, Campos EMS. Atenção primária, planejamento e modelos de atenção à saúde: Um enfoque sobre o plano diretor da Atenção Primária à Saúde de Minas Gerais. Rev APS. 2013;16(2):180-6.

28. Maciel FJ, Januário GC, Henriques CMA, Esteves CC, Silva MA et al. Indicadores de saúde auditiva em Minas Gerais - um estudo por macrorregião. ACR. 2013;18(4):275-84. http://dx.doi.org/10.1590/S2317-64312013000400008.

29. Cruz MS, Lima MCP, Santos JLF, Duarte YAO, Lebrão ML, Ramos-Cerqueira ATA. Uso do aparelho de amplificação sonora individual por idosos: estudo SABE - saúde, bem-estar e envelhecimento. ACR. 2013;18(2):133-42. http:// dx.doi.org/10.1590/S2317-64312013000200012.

Recebido em: Fev. 21, 2017 Aprovado em: Ago. 02, 2018 